\title{
Role and mechanisms of microRNA-503 in drug resistance reversal in HepG2/ADM human hepatocellular carcinoma cells
}

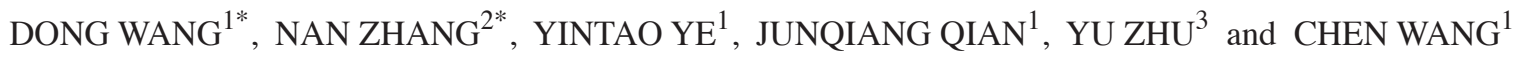 \\ ${ }^{1}$ Tianjin Medical University Cancer Institute and Hospital, National Clinical Research Center of Cancer, \\ Key Laboratory of Cancer Prevention and Therapy, Tianjin 300060; ${ }^{2}$ Department of Pharmacy, \\ Tianjin Children's Hospital, Tianjin 300074; ${ }^{3}$ Department of Clinical Laboratory, Tianjin Huan Hu Hospital, \\ Tianjin Key Laboratory of Cerebral Vessels and Neural Degeneration, Tianjin 300060, P.R. China
}

Received August 31, 2013; Accepted May 15, 2014

DOI: $10.3892 / \mathrm{mmr} .2014 .2591$

\begin{abstract}
Hepatocellular carcinoma is one of the most common malignancies worldwide and drug resistance is a major cause of treatment failure. In order to investigate the effects and mechanisms of microRNA-503 (miR-503) in the reversal of Adriamycin ${ }^{\circledR}(\mathrm{ADM})$ resistance in the drug-resistant HepG2/ADM hepatocellular cancer cell line, an ADM-resistant HepG2/ADM cell line was established using continuous drug exposure. HepG2/ADM cells overexpressing miR-503 were further established. HepG2/ADM cells overexpressing miR-503 demonstrated an enhanced sensitivity to ADM. Furthermore, miR-503 overexpression was found to increase intracellular rhodamine-123 levels and the rate of apoptosis, block the cell cycle at $\mathrm{G}_{0} / \mathrm{G}_{1}$-phase and significantly decrease intracellular superoxide dismutase and glutathione levels. The expression of a number drug resistance-related proteins, including multidrug resistance 1 , multi drug resistance-associated protein 1 , DNA excision repair protein ERCC-1, survivin and B-cell lymphoma 2, was significantly downregulated by miR-503 overexpression, as indicated by western blotting and a quantitative polymerase chain reaction. By contrast, levels of RhoE were increased. In addition, the phosphorylation of Akt was decreased and expression of cyclin-dependent kinase 1 was decreased by miR-503 overexpression. Furthermore, the secretion of transforming growth factor- $\beta$, interleukin (IL)- 6 and IL- 8 was downregulated, and the transcriptional activities of nuclear factor $\kappa$-light-chain-enhancer of activated B cells and activating protein-1 were significantly reduced. In conclusion, miR-503 was observed to reverse ADM resistance in HepG2/ADM cells by
\end{abstract}

Correspondence to: Mr. Chen Wang, Tianjin Medical University Cancer Institute and Hospital, National Clinical Research Center of Cancer, Key Laboratory of Cancer Prevention and Therapy, Huan-Hu-Xi Road, Tianjin 300060, P.R. China

E-mail: wangchen_tmutj@126.com

*Contributed equally

Key words: hepatocellular carcinoma, miR-503, drug resistance reversal inhibiting drug efflux, downregulating the expression of drug resistance-related proteins, blocking the cell cycle and promoting cell apoptosis.

\section{Introduction}

Hepatocellular carcinoma is one of the most common malignancies globally. Liver cancer causes $\sim 560,000$ mortalities each year, with half of these lethal cases reported in China (1). Hepatocellular carcinoma exhibits a subtle onset and its early symptoms are often undetectable. Therefore, the majority of patients with hepatocellular carcinoma are diagnosed in the middle or advanced stages of the disease. Patients with advanced stage hepatocellular carcinoma are not suitable candidates for surgical resection; therefore, they rely on alternative treatments, including hepatic artery intervention therapy, systematic drug therapy or organ transplantation. In recent years, the optimization of available treatment options and the development of a variety of targeted drugs have significantly enhanced the efficacy of current treatment and the survival of patients with liver cancer. However, certain problems remain, including liver cancer cell drug resistance (2). Adriamycin ${ }^{\circledR}$ (ADM) is an anti-tumor drug that belongs to the anthraquinone tetracyclic family. ADM inhibits DNA synthesis and transcription through targeting topoisomerase II, preventing cell growth and ultimately leading to tumor cell apoptosis. ADM is a key component of the transcatheter arterial chemoembolization and systemic chemotherapy programs (3). During long-term chemotherapy, liver cancer cells often develop resistance to ADM, causing a significant decrease in treatment efficacy. Therefore, investigating the mechanism underlying tumor cell drug resistance and developing novel methods to reverse drug resistance are of clinical significance for improving the treatment outcome and survival rate of patients. Previous studies have identified several mechanisms by which tumor cells develop drug resistance, including reducing drug concentration, increasing drug efflux through ATP-binding cassette $(\mathrm{ABC})$ transport proteins, enhancing anti-cancer drug clearance through the glutathione (GSH) system and downregulating the rate of apoptosis through the modulation of apoptotic pathways (4-7). 
micro RNAs (miRNAs) are endogenous non-protein-coding small RNAs, with a length of between 18 and 24 nucleotides. miRNAs bind to complementary sequences in target mRNAs, causing degradation of the target mRNA or gene silencing, thereby regulating gene expression at a post-transcriptional level (8). Evidence suggests that an association may exist between the expression of certain miRNAs and cancer. These studies have shown that, through regulating the expression of multiple target genes, a single miRNA molecule is capable of controlling cell growth and apoptosis and the migration and invasion of tumor cells, as well as tumor cell sensitivity to drug therapies $(9,10)$.

The role of miRNA-503 (miR-503) in cancer treatment has attracted significant attention. Studies have shown that the expression of miR-503 is decreased in oral and liver cancer $(11,12)$, but increased in parathyroid and adrenal cortical carcinomas $(13,14)$. Furthermore, in patients with adrenal carcinoma, an increase in miR-503 was correlated with reduced overall survival (13). In the HCCLM3 hepatoma cell line, miR-503 has been reported to arrest the cell cycle at $\mathrm{G}_{1}$-phase and inhibit cell migration and invasion (11). However, further studies are required to elucidate the role of miR-503 in drug resistance in liver cancer cells.

\section{Materials and methods}

Cell culture and stable cell line construction. The present study was approved by the ethics committee of Tianjin Medical University Cancer Institute and Hospital (Tianjin, China). The HepG2 human hepatocellular carcinoma cell line was purchased from the Cell Bank of the Chinese Academy of Science (Shanghai, China). Cells were cultured in medium containing $90 \%$ Eagle's Minimal Essential Medium and $10 \%$ fetal bovine serum at $37^{\circ} \mathrm{C}$ in $5 \% \mathrm{CO}_{2}$ with saturated humidity. Trypsin-EDTA $(0.25 \%)$ was used for cell digestion and passage. Cells in the logarithmic growth phase were used for all experiments. HepG2 cells were treated with low-dose ADM ( $\left.\mathrm{IC}_{50}, 1 / 10\right)$. Following the stabilization of the cell growth rate, the concentration of ADM was gradually increased in order to generate an ADM-resistant HepG2/ADM cell line.

miR-503 negative control miRNA mimics (Ago-miR-NC, 5'-GCTAGCCTGGACCACCCAGACCAACCTCGAGCA GAGGGCTTACTATCATTCTTCG-3') and stabilized miRNAs (Ago-miR-503, 5'-GTCGTATCCAGTGCAGGG TCCGAGGTGCACTGGATACGACCTGCAG-3') were purchased from Guangzhou RiboBio Co., Ltd., (Guangzhou, China) and transfected into cells using Lipofectamine ${ }^{\circledR} 2000$. The cell lines overexpressing miR-503 were established and further assessed using quantitative polymerase chain reaction (qPCR) analysis. The cells were divided into four experimental groups: i) HepG2 cells (parent group), ii) HepG2/ADM cells (control group), iii) HepG2/ADM negative control miRNA mimic-transfected cells as a negative control (Ago-miR-NC group) and iv) HepG2/ADM miRNA-transfected cells (Ago-miR-503 group).

MTS detection of the effect of miR-503 on tumor cell drug resistance. Cells were cultured on 96-well plates at a density of $2 \times 10^{4} / \mathrm{ml}$ with $100 \mu \mathrm{l}$ medium/well overnight, followed by the addition of $0,0.1,1,10$ and $100 \mu \mathrm{g} / \mathrm{ml} \mathrm{ADM}$ for $48 \mathrm{~h}$. A total of $100 \mu \mathrm{l}$ RPMI-1640 containing $0.5 \mathrm{mg} / \mathrm{ml}$ MTS was then added and the cells were cultured for a further $4 \mathrm{~h}$. Optical density (OD) values were detected at a wavelength of $490 \mathrm{~nm}$ using an enzyme-labeled meter. The growth inhibitory rate of ADM was calculated using the following formula: Rate of inhibition rate $=(1-$ OD value of the experimental group/OD value of the control group) x $100 \%$. Inhibition curves for different concentrations of ADM were plotted using drug concentration as the horizontal axis and inhibitory rate as the vertical axis. $\mathrm{IC}_{50}$ values were calculated using the following formula: Reversal fold $(\mathrm{RF})=\mathrm{IC}_{50}$ (without $\mathrm{miR}-503) / \mathrm{IC}_{50}$ (with miR-503).

Flow cytometry to assess rhodamine-123 (Rh-123) concentration, apoptosis and cell cycle in HepG2/ADM cells

$R h-123$ concentration. Cells were cultured in six-well plates at a density of $3 \times 10^{6} / \mathrm{ml}$ in $100 \mu \mathrm{l}$ medium/well overnight. The cells were then collected and washed, followed by the addition of $10 \mu \mathrm{M}$ Rh-123 dye solution for $1 \mathrm{~h}$. The cell density was adjusted to $1 \times 10^{6} / \mathrm{ml}$ and the fluorescence intensity of $\mathrm{Rh}-123$ excited at $488 \mathrm{~nm}$ was detected using flow cytometry in order to measure the intracellular concentration of Rh-123.

Cell cycle distribution. Cells were cultured in six-well plates at a density of $3 \times 10^{6} / \mathrm{ml}$ in $100 \mu \mathrm{l}$ medium/well overnight. Cells were then collected and washed, prior to the addition of $100 \mu \mathrm{l}$ RNase-A and propidium iodide (PI) for $30 \mathrm{~min}$. The cell density was adjusted to $1 \times 10^{6} / \mathrm{ml}$ and the fluorescence intensity of PI excited at $488 \mathrm{~nm}$ was detected using flow cytometry to assess the cell cycle distribution of the cells.

Detection of apoptosis. Cells were cultured in six-well plates at a density of $3 \times 10^{6} / \mathrm{ml}$ in $100 \mu 1$ medium/well overnight, followed by treatment with $3 \mu \mathrm{g} / \mathrm{ml}$ ADM for a further $24 \mathrm{~h}$. Cells were then collected, washed, and PI/Annexin V-fluorescein isothiocyanate (FITC) was added to the cultured cells for $15 \mathrm{~min}$. The cell density was adjusted to $1 \times 10^{6} / \mathrm{ml}$ and the fluorescence intensity of Annexin V-FITC and PI excited at $488 \mathrm{~nm}$ was detected using flow cytometry to assess cell apoptosis.

Biochemical assays to detect superoxide dismutase (SOD) and GSH activity. Cells were lysed and SOD activity was detected using the nitroblue tetrazolium method. GSH activity was examined by measuring the absorbance of the yellow 5-thio-2-nitrobenzoic acid and glutathione disulfide generated from the 5,5'-dithiobis-(2-nitrobenzoic acid) reaction. The experiments were performed in accordance with the kit manufacturer's instructions (Beyotime Institute of Biotechnology, Haimen, China) and absorbance was measured using a microplate reader.

Liquidchip assay to detect drug resistance-related cytokines. Cells were cultured in six-well plates at a density of $3 \times 10^{6} / \mathrm{ml}$ in $100 \mu \mathrm{lmedium} /$ well overnight and then incubated with fresh serum-free culture medium for a further $24 \mathrm{~h}$. The supernatant was collected and the levels of transforming growth factor (TGF)- $\beta$, interleukin (IL)- 6 and IL- 8 were determined using liquidchip assay (EMD Millipore, Billerica, MA, USA) according to the manufacturer's instructions. 
Western blot analysis to detect the protein expression of multidrug resistance genes in HepG2/ADM cells. Cells were lysed and total proteins were extracted. Total protein was quantified using the bicinchoninic acid method. A total of $80 \mu \mathrm{g}$ protein was separated using $12 \%$ SDS-PAGE and transferred to polyvinylidene fluoride (PVDF) membranes. The membranes were then blocked using $10 \%$ milk and incubated with the following monoclonal antibodies: Anti-multidrug resistance 1 (MDR1) (diluted 1:600), -multidrug resistance-associated protein 1 (MRP 1) (diluted 1:600), -DNA excision repair protein ERCC-1 (ERCC1) (diluted 1:600), -RhoE (diluted 1:500), -survivin (diluted 1:800), -B-cell lymphoma-2 (Bcl-2) (diluted 1:500), -phosphorylated cyclin-dependent kinase 1 (P-CDK1) (diluted 1:400) and -phosphorylated Akt (diluted 1:400), all from Santa Cruz Biotechnology, Inc. (Santa Cruz, CA, USA), and anti- $\beta$-actin (diluted 1:5,000) from Sigma-Aldrich (St. Louis, MO, USA) at $4^{\circ} \mathrm{C}$ overnight. The PVDF membranes were then incubated with horseradish peroxidase-conjugated secondary antibodies at room temperature for $2 \mathrm{~h}$. Immunoreactive bands were detected using an enhanced chemiluminescence kit following washing. $\beta$-actin was used as an internal control.

qPCR analysis to detect the mRNA expression of multidrug resistance genes in HepG2/ADM cells. Total RNA was extracted using TRIzol ${ }^{\circledR}$ reagent and qPCR was performed using a commercial kit and the following primers: MDR1, 5'-AAAAAGATCAACTCGTACCACTC-3' (forward), 5'-GCACAAAATACACCAACAA-3' (reverse); MRP1, 5'-ACTTCCACATCTGCTTCGTCAGTG-3' (forward), 5'-ATTCAGCCACAGGAGGTAGAGAGC-3' (reverse); ERCC1, 5'-GGGAATTTGGCGACGTAATTC-3' (forward), 5'-GCGGAGGCTGAGGAACAG-3' (reverse); RhoE, 5'-CCT CCACGTTGATTCGACTGTT-3' (forward), 5'-TGTAAAAGC CGTACGTTGCGGT-3' (reverse); survivin, 5'-GCATGGGTG CCCCGACGTTG-3' (forward), 5'-GCTCCGGCCAGAGGC CTCAA-3' (reverse); Bcl-2, 5'-ACGGGGTGAACTGGGGGA GGA-3' (forward), 5'-TGTTTGGGGCAGGCATGTTGA CTT-3' (reverse); and $\beta$-actin, 5'-TGAGCGCGGCTACAG CTT-3' (forward), 5'-TCCTTAATGTCACGCACGATTT-3' (reverse). The PCR conditions were as follows: Denaturation at $94^{\circ} \mathrm{C}$ for $3 \mathrm{~min}$ followed by 40 cycles of amplification at $95^{\circ} \mathrm{C}$ for $5 \mathrm{sec}, 65^{\circ} \mathrm{C}$ for $35 \mathrm{sec}$ and $72^{\circ} \mathrm{C}$ for $60 \mathrm{sec}$, then a final extension for $5 \mathrm{~min}$ at $72^{\circ} \mathrm{C}$. $\beta$-actin was used as a internal control.

Double fluorescent reporter gene assay to detect the transcriptional activity of $N F-\kappa B$ and AP-1. Cells were cultured in six-well plates at a density of $3 \times 10^{6} / \mathrm{ml}$ in $100 \mu \mathrm{l}$ medium/well overnight. A total of $0.1 \mu \mathrm{g}$ pGL4.74 [hRluc/TK] and $0.1 \mu \mathrm{g}$ pGL 4.32 [luc2P/NF-kB-RE/Hypro] or pGL 4.44 [luc2P/AP-1-RE/Hygro] plasmids (Jinxing BioTech, Tianjin, China) were then added into each well and transfected into the cells according to the manufacturer's instructions. The cells were then cultured for $24 \mathrm{~h}$, washed and changed into fresh medium. A Dual-Glo ${ }^{\mathrm{TM}}$ Luciferase assay system (Promega Corporation, Madison, WI, USA) was used to detect the activity of the two fluorescent enzymes.

Statistical analysis. Experimental data are presented as the mean \pm standard deviation. SPSS 13.0 software (SPSS, Inc., Chicago, IL, USA) was used to conduct the statistical

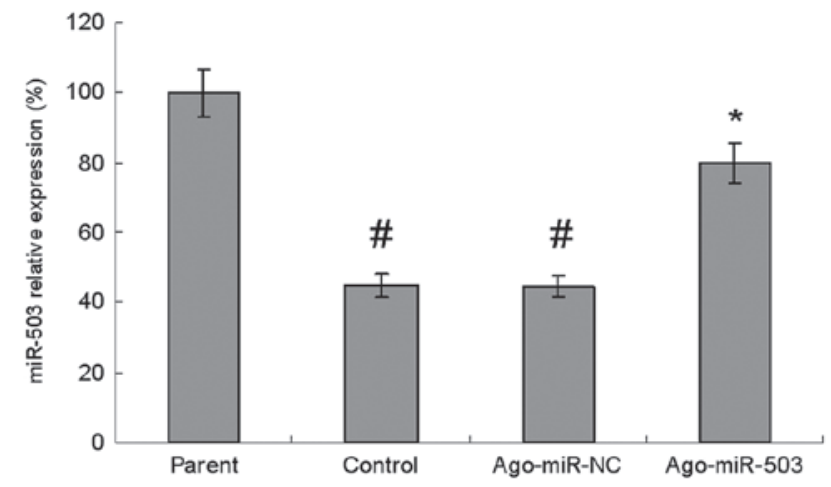

Figure 1. miR-503 expression in HepG2 and HepG2/ADM cells, as determined using quantitative polymerase chain reaction analysis. Values are presented as the mean \pm standard deviation $(n=5) .{ }^{*} \mathrm{P}<0.05$ versus the parent group; ${ }^{*} \mathrm{P}<0.05$ versus the control group. ADM, Adriamycin; miR-503, microRNA-503; NC, negative control.

analysis of data. One-way analysis of variance was performed to compare the results. A value of $\mathrm{P}<0.05$ was considered to indicate a statistically significant difference.

\section{Results}

Establishment of the miR-503 overexpressing HepG2/ADM cell line. miR-503 expression in HepG2 and HepG2/ADM cells was measured using qPCR. miR-503 expression was decreased in the HepG2/ADM cells compared with that in the parent group, while in the miR-503-overexpressing HepG2/ADM cells miR-503 expression was increased relative to the expression in the control and Ago-miR-NC groups (Fig. 1).

miR-503 increases ADM sensitivity, promotes apoptosis, arrests the cell cycle and increases intracellular Rh-123 concentrations in HepG2/ADM cells. HepG2/ADM cells were established through long-term culture in media containing ADM. MTS assay revealed that HepG2/ADM cells showed enhanced resistance to ADM compared with the parent group. $\mathrm{IC}_{50}$ values were calculated to assess $\mathrm{ADM}$ resistance. The ADM resistance of the HepG2/ADM cell line $\left(\mathrm{IC}_{50}, 11.2 \mu \mathrm{g} / \mathrm{ml}\right)$ was found to be 62.2 -fold that of the parent HepG2 cell line $\left(\mathrm{IC}_{50}, 0.18 \mu \mathrm{g} / \mathrm{ml}\right)$.

The MTS assay results further revealed that the sensitivity of the Ago-miR-503-transfected cells to ADM was significantly increased compared with that of the control and Ago-miR-NC groups. Based on the $\mathrm{IC}_{50}$ value of Ago-miR-503, which was $3.3 \mu \mathrm{g} / \mathrm{ml}$, the RF was 3.4. An increase in apoptosis and cell cycle arrest at $\mathrm{G}_{0} / \mathrm{G}_{1}$-phase were also observed in the Ago-miR-503 group as compared with the control and Ago-miR-NC groups. Furthermore, the intracellular Rh-123 content was found to be increased in the Ago-miR-503 group compared with that in the control and Ago-miR-NC groups, suggesting that miR-503 reversed ADM resistance through increasing intracellular drug concentration (Fig. 2).

miR-503 decreases the expression of MDR1, MRP1, ERCC1, survivin and $B C l-2$, decreases the activity of $S O D$ and $G S H$, and increases the expression of RhoE in HepG2/ADM cells. In order to investigate the mechanism underlying the 


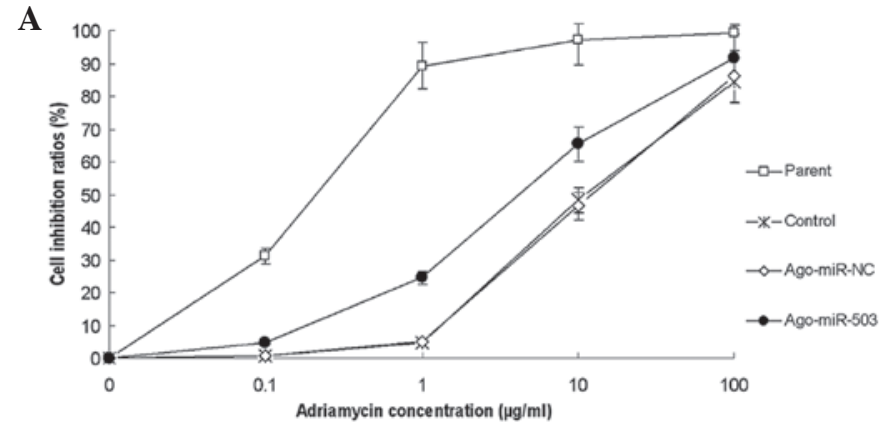

B
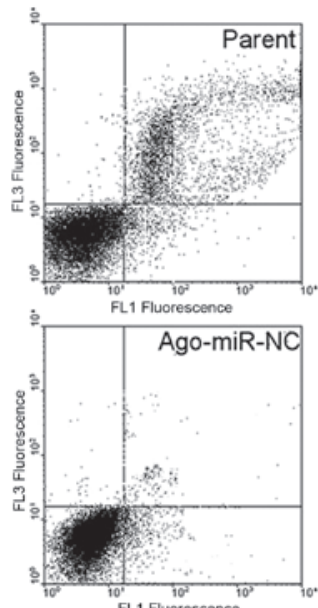

FL1 Fluorescence

\section{C}
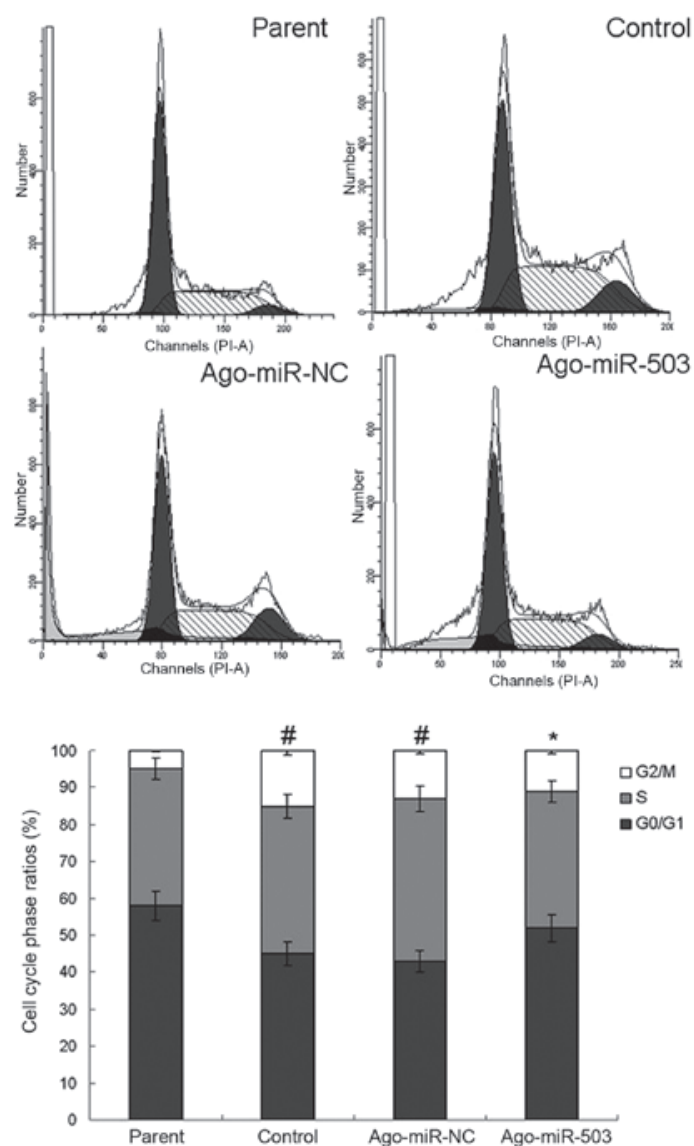

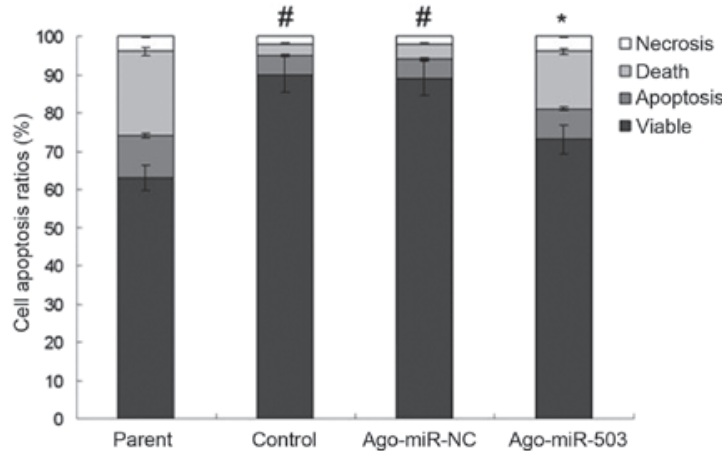

D
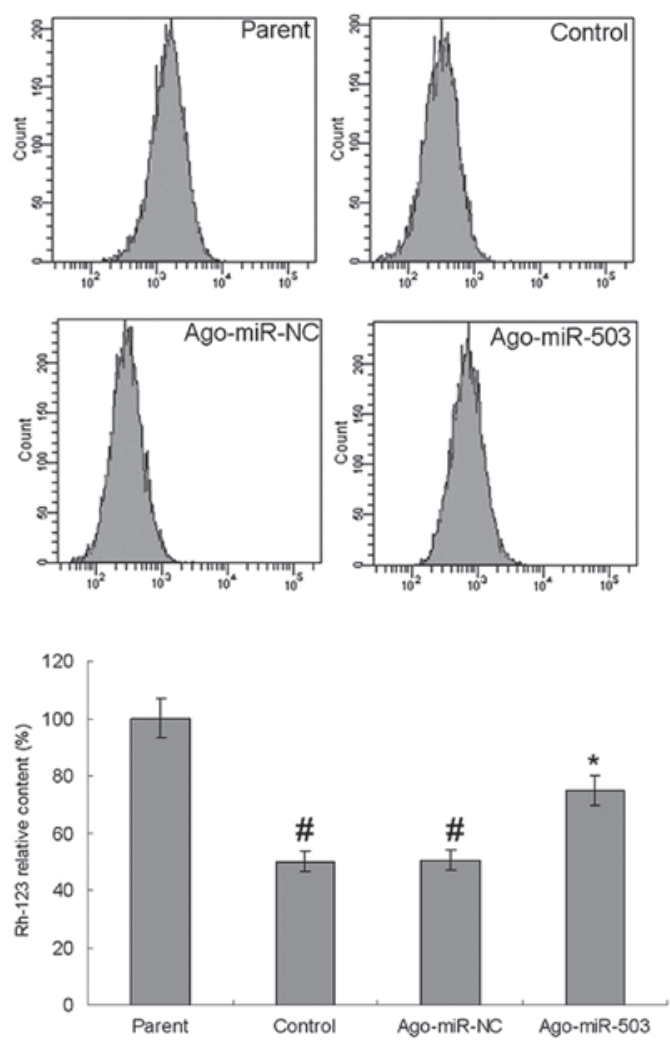

Figure 2. Effect of miR-503 on the reversal of ADM resistance in human hepatocellular carcinoma HepG2/ADM cells. (A-D) Effect of miR-503 on (A) ADM sensitivity, determined using MTS assay $(n=5) ;(B)$ apoptosis, determined using flow cytometry $(n=3) ;(C)$ cell cycle, determined using flow cytometry ( $\mathrm{n}=3$ ) and (D) intracellular Rh-123 concentrations, determined using flow cytometry ( $=3$ ), in HepG2/ADM cells. Values are presented as the mean \pm standard deviation. ${ }^{\#} \mathrm{P}<0.05$ versus the parent group; ${ }^{*} \mathrm{P}<0.05$ versus the control group. ADM, Adriamycin; Rh, rhodamine; miR-503, microRNA-503; NC, negative control. 
A

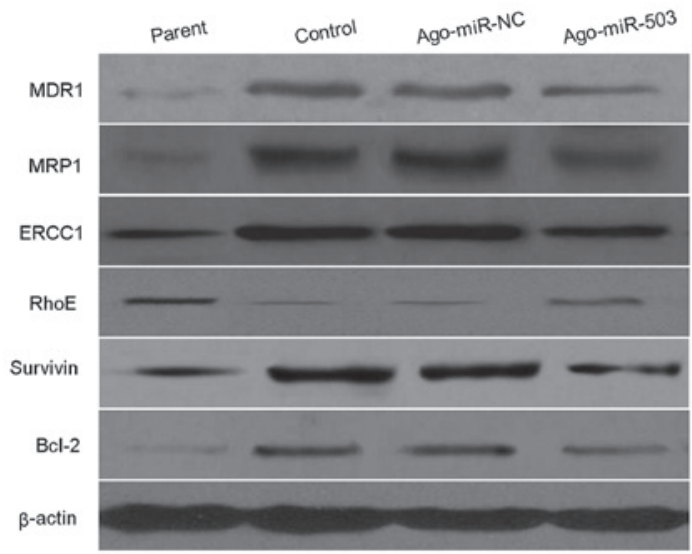

C

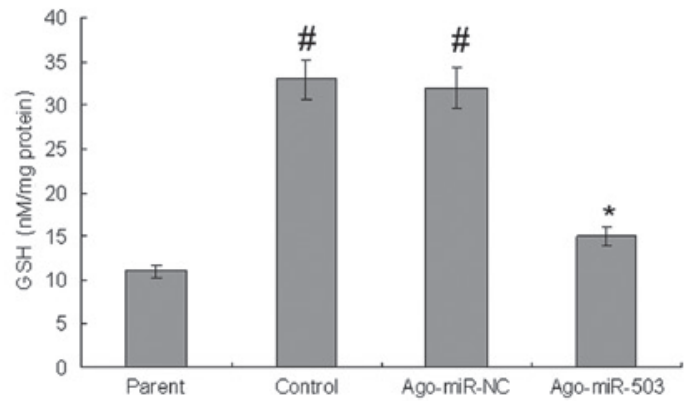

B
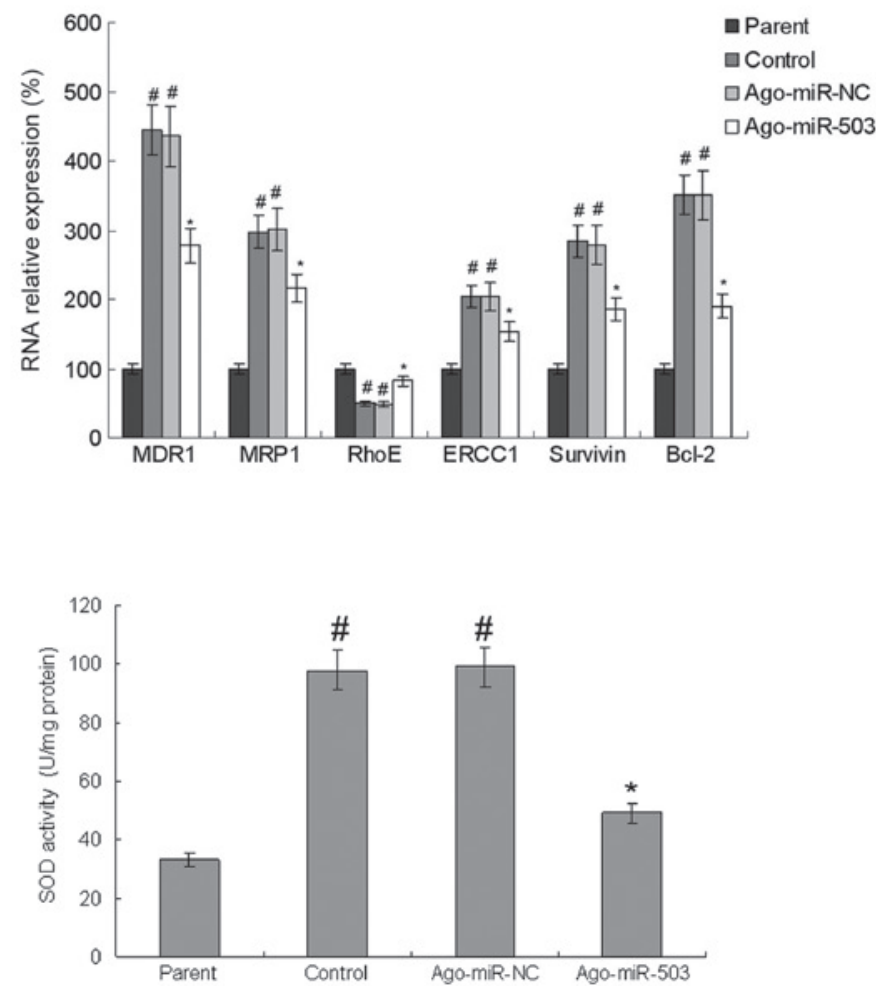

Figure 3. Effect of miR-503 on the expression of drug resistance genes in human hepatocellular carcinoma HepG2/ADM cells. (A and B) Effect of miR-503 on (A) protein and (B) mRNA expression of drug resistance genes in HepG2/ADM cells, determined using western blot analysis and quantitative polymerase chain reaction analysis, respectively. $\beta$-actin expression was used as an internal control. (C) Effect of miR-503 on SOD and GSH activity in HepG2/ADM cell lines, determined using biochemical assay. Values are presented as the mean \pm standard deviation $(n=5) .{ }^{*} \mathrm{P}<0.05$ versus the parent group; ${ }^{*} \mathrm{P}<0.05$ versus the control group. ADM, Adriamycin; MDR1, multidrug resistance 1; MRP1, multidrug resistance-associated protein 1; ERCC1, DNA excision repair protein ERCC-1; Bcl-2, B-cell lymphoma-2; SOD, superoxide dismutase; GSH, glutathione; miR-503, microRNA-503; NC, negative control.

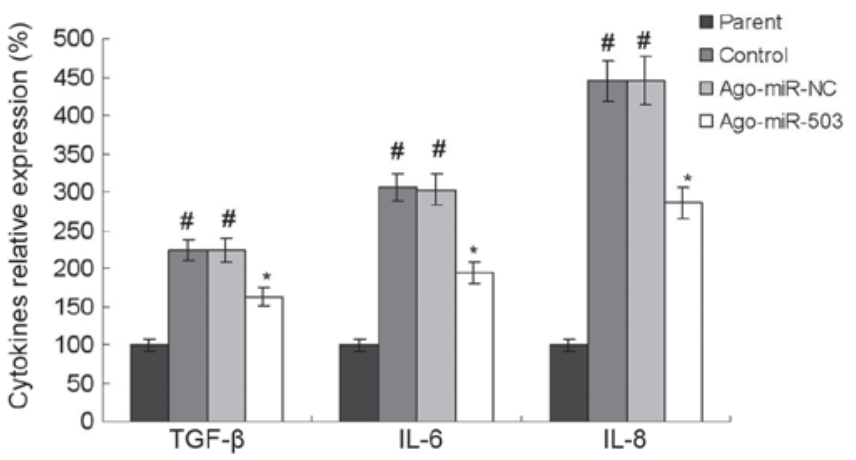

Figure 4. Effect of miR-503 on the production of cytokines associated with ADM resistance in human hepatocellular carcinoma HepG2/ADM cell lines. The effect of miR-503 on the expression of TGF- $\beta$ and IL- 6 and -8 in HepG2/ADM cell lines was determined using liquidchip assay. Values are presented as the mean \pm standard deviation $(\mathrm{n}=5) .{ }^{*} \mathrm{P}<0.05$ versus the parent group; ${ }^{*} \mathrm{P}<0.05$ versus the control group. ADM, Adriamycin; TGF- $\beta$, transforming growth factor- $\beta$; IL, interleukin; miR-503, microRNA-503; NC, negative control.

miR-503-induced reversal of ADM resistance and promotion of apoptosis in HepG2/ADM cells, the expression of relevant genes was detected. The expression of MDR1 and MRP1, two multidrug resistance proteins, was observed to be significantly reduced in the Ago-miR-503 cells. The activity of the small detoxifying molecules SOD and GSH was also found to be significantly reduced. Furthermore, the expression of ERCC1, a protein involved in repairing DNA damage, and survivin and Bcl-2, which inhibit apoptosis, was also observed to be significantly downregulated by miR-503. Of note, miR-503 was found to increase RhoE expression in cells in the Ago-miR-503 group as compared with those in the control and Ago-miR-NC groups. qPCR revealed that the regulatory role of miR-503 on the aforementioned proteins was achieved through transcriptional regulation (Fig. 3).

miR-503 inhibits the secretion of TGF- $\beta$ and IL-6 and -8 in HepG2/ADM cells. Cytokines have an important role in the initiation, development and progression of tumors (15). In the present study, the expression of three cytokines secreted by HepG 2 cells, TGF- $\beta$ and IL- 6 and -8 , was analyzed. Compared with the control and Ago-miR-NC groups, the expression of TGF- $\beta$ and IL- 6 and -8 in the Ago-miR-503 group was significantly decreased (Fig. 4).

miR-503 inhibits Akt phosphorylation, increases CDKI expression and inhibits the transcriptional activity of $N F-\kappa B$ and AP-1 in HepG2/ADM cells. In order to study the molecular mechanism underlying miR-503-induced $\mathrm{G}_{0} / \mathrm{G}_{1}$-phase arrest in HepG2/ADM cells, the phosphoinositide 3-kinase (PI3K)/Akt and cyclin/CDK1 signaling pathways, which are closely associated with the cell cycle, were analyzed. Western blot analysis revealed that Akt phosphorylation was decreased 

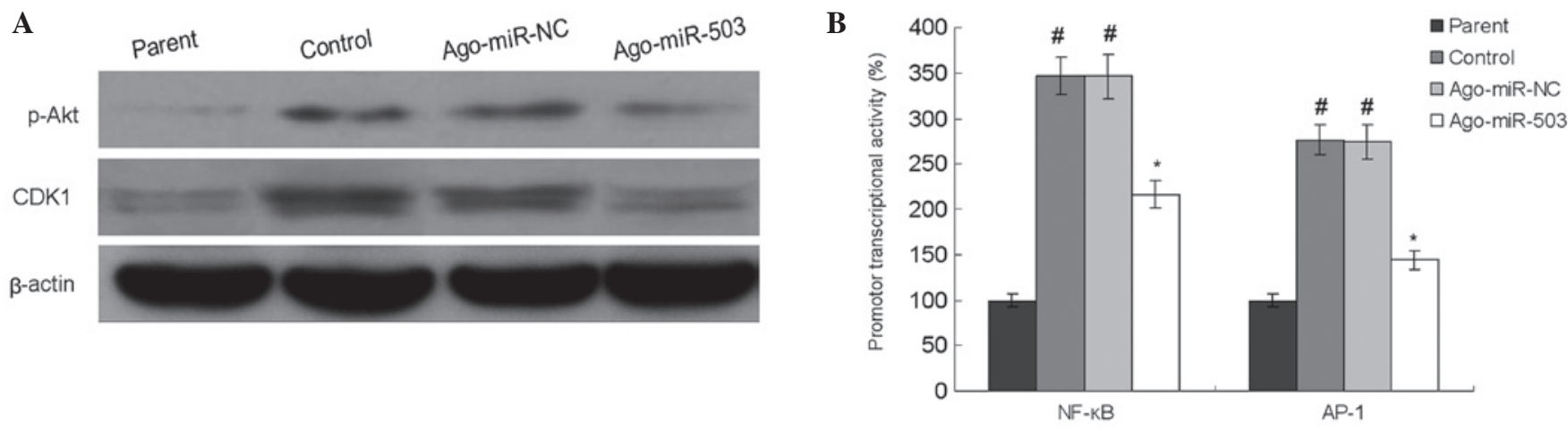

Figure 5. Effect of miR-503 on ADM resistance-related signaling molecules in HepG2/ADM human hepatocellular carcinoma cells. (A) Effect of miR-503 on Akt phosphorylation in HepG2/ADM cells, determined using western blot analysis. $\beta$-actin was used as an internal control. (B) Effect of miR-503 on the transcriptional activity of NF- $\mathrm{kB}$ and AP-1 in HepG2/ADM cells, determined using double fluorescent reporter gene assay. Values are presented as the mean \pm standard deviation $(\mathrm{n}=5) .{ }^{~} \mathrm{P}<0.05$ versus the parent group; ${ }^{*} \mathrm{P}<0.05$ versus the control group. ADM, Adriamycin; NF- $\mathrm{kB}$, nuclear factor $\kappa$-light-chain-enhancer of activated B cells; AP-1, activator protein 1; CDK1, cyclin-dependent kinase 1; p-Akt, phosphorylated Akt; NC, negative control; miR-503, microRNA-503.

in the Ago-miR-503 group, indicating that the PI3K/Akt pathway was inhibited. The expression of CDK1 was also decreased, indicating that the cyclin/CDK1 signaling pathway was also inhibited by miR-503. In order to elucidate the molecular mechanism underlying the regulation of cytokine secretion by miR-503, gene reporter systems were used to evaluate the transcriptional activity of NF- $\mathrm{KB}$ and AP-1. The transcriptional activities of these two transcription factors were significantly decreased (Fig. 5).

\section{Discussion}

Increasing drug efflux and reducing drug concentration are major mechanisms by which tumor cells develop resistance to chemotherapeutic drugs. $\mathrm{ABC}$ family transmembrane transporter proteins, including MDR1 and MRP1, pump drugs out of cells and are often overexpressed in drug-resistant tumor cells (16). Upon miR-503 transfection, flow cytometry revealed that the concentration of intracellular Rh-123 was increased. This finding indirectly indicates that the mechanism by which miR-503 reverses drug resistance in HepG2/ADM cells may be associated with reducing drug efflux. Furthermore, miR-503 was found to decrease the protein and mRNA expression of MDR1 and MRP1, providing evidence for the above hypothesis.

In addition to MDR1 and MRP1, other molecules may also be involved in ADM resistance in tumor cells. ERCC1 repairs DNA damage induced by chemotherapeutic drugs (17); therefore, its expression may be associated with HepG2/ADM cell drug resistance. Western blot analysis revealed that ERCC1 protein expression was high in HepG2/ADM cells, but decreased significantly following miR-503 treatment. This decrease in expression was associated with reduced mRNA expression. SOD and GSH act as detoxifying molecules in tumor tissues and reduce the toxicity of the chemotherapeutic drugs $(18,19)$. The present study showed that the SOD and GSH levels in HepG2/ADM cells were significantly increased compared with those in the normal HepG2 cells. Subsequent to reversing the drug resistance in HepG2/ADM cells using miR-503, the SOD and GSH levels decreased, indicating that these two molecules may also have a role in the drug resistance mechanisms of tumor cells. RhoE is an atypical member of the Rho GTPase family and is present in a constitutively active state for GTP-binding. RhoE expression has recently been found to be decreased in certain major liver cancer cell lines and tissues; this decreased expression may in turn promote tumor invasion and metastasis (20). In the present study miR-503 was found to increase the expression of RhoE, indicating that miR-503 plays an inhibitory role in tumor invasion and metastasis.

Survivin is a member of the anti-apoptotic gene family. This gene exhibits certain tumor-specific characteristics, and is solely expressed in tumors and embryonic tissues. Survivin can inhibit tumor apoptosis and promote proliferation and angiogenesis. Therefore, it is considered to be a highly valuable therapeutic target for tumor treatment $(21,22)$. In the present study, miR-503 was found to decrease survivin expression, which is consistent with the observation that miR-503 increased the rate of tumor cell apoptosis. Bcl-2 has important functions in apoptosis and inhibits tumor cell apoptosis. The overexpression of $\mathrm{Bcl}-2$ has been found to be associated with drug resistance in numerous tumor cells $(23,24)$. In the non-small-cell cisplatin-resistant A549/CDDP cell line, miR-503 has been reported to reduce the expression of Bcl-2 and reverse drug resistance (25). In the present study Bcl-2 expression was observed to be significantly increased in HepG2/ADM cells, indicating that the anti-apoptotic effect of Bcl-2 may be one of the mechanisms by which drug resistance develops in tumor cells. In the present study, miR-503 transfection significantly reduced Bcl-2 expression in HepG2/ADM cells, which is consistent with the enhanced apoptosis detected using flow cytometry. This may be one of the important mechanisms underlying the miR-503-induced reversal of drug resistance in tumor cells.

In the present study, the overexpression of miR-503 was found to arrest HepG2/ADM cells at $\mathrm{G}_{0} / \mathrm{G}_{1}$-phase. CDK1 is an important regulatory protein in the cell cycle and has an essential role in $\mathrm{G}_{0} / \mathrm{G}_{1^{-}}$to $S$-phase progression (26). In tumor cells, checkpoint cyclins often become abnormally regulated by CDKs, resulting in uncontrolled tumor cell proliferation $(26,27)$. The present study found that miR-503 decreased CDK1 expression. In HepG2/ADM cells, miR-503 may regulate CDK1 through the same mechanism. PI3K/Akt is another signaling pathway that plays an important role in regulating the cell cycle (28). In the present study, miR-503 was observed to 
inhibit Akt phosphorylation and, in turn, inhibit the activity of the PI3K/Akt signaling pathway, thereby inhibiting the proliferation of tumor cells and reversing tumor cell drug resistance.

Cytokines have an important role in the occurrence and development of liver cancer (29,30). TGF- $\beta$ and IL- 6 and -8 expression was observed in normal HepG2 cells; however, in HepG2/ADM cells, the concentration of these cytokines was significantly increased. These cytokines may help liver cancer cells to regulate their microenvironment, enhance their proliferation, invasion and metastasis, and ultimately survive in the challenging environment (30). The present study showed that miR-503 downregulated the expression of TGF- $\beta$ and IL- 6 and -8 . NF- $\kappa$ B and AP-1 are two important transcription factors that have key roles in cell proliferation, apoptosis and the secretion of inflammatory cytokines in normal and tumor cells $(31,32)$. The present study indicated that miR-503 may negatively regulate the activity of NF- $\mathrm{kB}$ and AP-1. Although the specific mechanism requires further investigation, this negative regulation may be one of the mechanisms responsible for the miR-503-induced reversal of drug resistance in HepG2/ADM cells.

In conclusion, we considered miR-503 as a potential target for the reversal of drug resistance in human hepatocellular carcinoma. The mechanisms underlying this miR-503-induced reversal of drug resistance may involve the inhibition of drug efflux, the downregulation of drug resistance-associated gene expression, the prevention of cell cycle progression and the promotion of cell apoptosis.

\section{References}

1. Nordenstedt H, White DL and El-Serag HB: The changing pattern of epidemiology in hepatocellular carcinoma. Dig Liver Dis 42 (Suppl 3): S206-S214, 2010.

2. Thomas MB and Zhu AX: Hepatocellular carcinoma: the need for progress. J Clin Oncol 23: 2892-2899, 2005.

3. Bruix J, Sala M and Llovet JM: Chemoembolization for hepatocellular carcinoma. Gastroenterology 127 (5 Suppl 1): S179-S188, 2004.

4. Szakács G, Paterson JK, Ludwig JA, et al: Targeting multidrug resistance in cancer. Nat Rev Drug Discov 5: 219-234, 2006.

5. Zhang K, Mack P and Wong KP: Glutathione-related mechanisms in cellular resistance to anticancer drugs. Int J Oncol 12 871-882, 1998.

6. Johnstone RW, Ruefli AA and Lowe SW: Apoptosis: a link between cancer genetics and chemotherapy. Cell 108: 153-164, 2002.

7. Rabik CA and Dolan ME: Molecular mechanisms of resistance and toxicity associated with platinating agents. Cancer Treat Rev 33: 9-23, 2007.

8. Calin GA and Croce CM: MicroRNA signatures in human cancers. Nat Rev Cancer 6: 857-866, 2006.

9. Gandellini P, Profumo V, Folini M and Zaffaroni N: MicroRNAs as new therapeutic targets and tools in cancer. Expert Opin Ther Targets 15: 265-279, 2011.

10. Trang P, Weidhaas JB and Slack FJ: MicroRNAs as potential cancer therapeutics. Oncogene 27 (Suppl 2): S52-S57, 2008.
11. Zhou J and Wang W: Analysis of microRNA expression profiling identifies microRNA-503 regulates metastatic function in hepatocellular cancer cell. J Surg Oncol 104: 278-283, 2011.

12. Lu YC, Chen YJ, Wang HM, et al: Oncogenic function and early detection potential of miRNA-10b in oral cancer as identified by microRNA profiling. Cancer Prev Res (Phila) 5: 665-674, 2012.

13. Özata DM, Caramuta S, Velázquez-Fernández $\mathrm{D}$, et al: The role of microRNA deregulation in the pathogenesis of adrenocortical carcinoma. Endocr Relat Cancer 18: 643-655, 2011.

14. Corbetta S, Vaira V, Guarnieri V, et al: Differential expression of microRNAs in human parathyroid carcinomas compared with normal parathyroid tissue. Endocr Relat Cancer 17: 135-146, 2010.

15. Bruchard M and Ghiringhelli F: Tumor microenvironment: regulatory cells and immunosuppressive cytokines. Med Sci (Paris) 30: 429-435, 2014 (In French).

16. Keppler D: Multidrug resistance proteins (MRPs, ABCCs): importance for pathophysiology and drug therapy. In: Drug Transporters: Handbook of Experimental Pharmacology. Fromm MF and Kim RB (eds). Springer, Heidelberg, pp299-323, 2011.

17. Hu JJ, de la Garza J, Srinivasan SK, et al: Important roles of ERCC1 in DNA repair and targeted therapy. In: Cancer Drug Discovery and Development. Panasci L, Aloyz R and Alaoui-Jamali M (eds). Springer, New York, NY, pp129-142, 2013.

18. Chen HH and Kuo MT: Role of glutathione in the regulation of Cisplatin resistance in cancer chemotherapy. Met Based Drugs 2010: 430939, 2010.

19. Shanker M, Willcutts D, Roth JA and Ramesh R: Drug resistance in lung cancer. Lung Cancer: Targets and Therapy 1: 23-36, 2010.

20. Grise F, Sena S, Bidaud-Meynard A, et al: Rnd3/RhoE is down-regulated in hepatocellular carcinoma and controls cellular invasion. Hepatology 55: 1766-1775, 2012.

21. Ryan BM, O'Donovan N and Duffy MJ: Survivin: A new target for anti-cancer therapy. Cancer Treat Rev 35: 553-562, 2009.

22. Altieri DC: Survivin and IAP proteins in cell-death mechanisms. Biochem J 430: 199-205, 2010.

23. Ma J, Zhu W and Zhou Q: Expression and significance of bag-1, bcl-2 in non-small cell lung cancer and the correlation with multi-drug resistance. Zhongguo Fei Ai Za Zhi 12: 1089-1094, 2009 (In Chinese).

24. Yip KW and Reed JC: Bcl-2 family proteins and cancer. Oncogene 27: 6398-6406, 2008.

25. Qiu T, Zhou L, Wang T, Xu J, Wang J, et al: miR-503 regulates the resistance of non-small cell lung cancer cells to cisplatin by targeting Bcl-2. Int J Mol Med 32: 593-598, 2013.

26. Malumbres $M$ and Barbacid M: Cell cycle, CDKs and cancer: a changing paradigm. Nat Rev Cancer 9: 153-166, 2009.

27. Sarkar S, Dey BK and Dutta A: miR-322/424 and -503 are induced during muscle differentiation and promote cell cycle quiescence and differentiation by down-regulation of Cdc25A. Mol Biol Cell 21: 2138-2149, 2010.

28. LoPiccolo J, Blumenthal GM, Bernstein WB and Dennis PA: Targeting the PI3K/Akt/mTOR pathway: effective combinations and clinical considerations. Drug Resist Updat 11: 32-50, 2008.

29. El-Serag HB and Rudolph KL: Hepatocellular carcinoma: epidemiology and molecular carcinogenesis. Gastroenterology 132: 2557-2576, 2007.

30. Budhu A and Wang XW: The role of cytokines in hepatocellular carcinoma. J Leukoc Biol 80: 1197-1213, 2006.

31. Gilmore TD: Introduction to NF-kappaB: players, pathways, perspectives. Oncogene 25: 6680-6684, 2006.

32. Shaulian E and Karin M: AP-1 in cell proliferation and survival. Oncogene 20: 2390-2400, 2001. 